\title{
Gradient computation for VTI acoustic wavefield tomography
}

Vladimir Li ${ }^{1}$, Hui Wang ${ }^{1}$, Ilya Tsvankin ${ }^{1}$, Esteban Díaz ${ }^{1}$ \& Tariq Alkhalifah ${ }^{2}$

${ }^{1}$ Center for Wave Phenomena, Colorado School of Mines

${ }^{2}$ King Abdullah University of Science and Technology

\section{SUMMARY}

Wavefield tomography can handle complex subsurface geology better than ray-based techniques and, ultimately, provide a higher resolution. Here, we implement forward and adjoint wavefield extrapolation for VTI (transversely isotropic with a vertical symmetry axis) media using a pseudospectral operator that employes a separable approximation of the P-wave dispersion relation. This operator is employed to derive the gradients of the differential semblance optimization (DSO) and modified stack-power objective functions. We also obtain the gradient expressions for the data-domain objective function, which can incorporate borehole information necessary for stable VTI velocity analysis. These gradients are compared to the ones obtained with a space-time finite-difference (FD) scheme for a system of coupled wave equations. Whereas the kernels computed with the two wave-equation operators are similar, the pseudospectral method is not hampered by the imprint of the shear-wave artifact. Numerical examples also show that the modified stack-power objective function produces cleaner gradients than the more conventional DSO operator.

\section{INTRODUCTION}

In accordance with the semblance principle (Al-Yahya, 1989), image-domain wavefield tomography enforces the consistency of migrated images for different experiments (Sava, 2014). The corresponding objective function usually involves evaluating the energy focusing in the extended domain (Rickett and Sava, 2002; Sava and Fomel, 2006; Sava and Vasconcelos, 2011), which can be done with DSO (Symes and Carazzone, 1991) or image-power estimates (Chavent and Jacewitz, 1995).

Anisotropic wavefield tomography is typically implemented under the pseudoacoustic assumption originally proposed by Alkhalifah (1998). Acoustic modeling in TI media can be performed with space-time FD schemes applied to coupled second-order equations (Duveneck et al., 2008; Fletcher et al., 2009; Zhang et al., 2011). Pseudospectral methods provide an alternative way to propagate only P-waves without the shearwave artifacts (Etgen and Brandsberg-Dahl, 2009; Du et al., 2014). Separable P-mode dispersion-relation approximations for TI media are described in Pestana et al. (2011), Du et al. (2014), and Schleicher and Costa (2015).

Weibull and Arntsen (2014) and Li et al. (2016) attempt to estimate the VTI parameters using different image-domain tomographic algorithms. Li et al. (2015) study the defocusing in the extended domain caused by errors in the VTI parameters. Here, we derive and test the wavefield-tomography gradients in the image and data domains using a wave-equation operator based on a separable P-mode approximation for VTI media.

\section{SEPARABLE P-MODE APPROXIMATION}

P-wave kinematics in VTI media is controlled by the vertical velocity $V_{P 0}$ and Thomsen parameters $\varepsilon$ and $\delta$ (Tsvankin, 2012). Alternative parameter combinations for acoustic VTI media also involve the horizontal velocity $\left[V_{\text {hor }}=V_{P 0} \sqrt{1+2 \varepsilon}\right]$, the anellipticity parameter $\eta=(\varepsilon-\delta) /(1+2 \delta)$, and the zerodip normal-moveout (NMO) velocity $\left[V_{\mathrm{nmo}}=V_{P 0} \sqrt{1+2 \delta}\right]$.

Pseudospectral methods are designed to evaluate the spatial wavefield derivatives in the wavenumber domain. Anisotropic extrapolation requires approximate dispersion relations with separable wavenumber and model-parameter terms. Employing the first-order Padé expansion, the P-wave separable dispersion relation for VTI media is obtained as (Schleicher and Costa, 2015):

$$
\begin{aligned}
\omega^{2} & =(1+2 \varepsilon) V_{P 0}^{2} k_{x}^{2}+V_{P 0}^{2} k_{z}^{2}-2(\varepsilon-\delta) V_{P 0}^{2} \frac{k_{x}^{2} k_{z}^{2}}{k_{x}^{2}+k_{z}^{2}} \\
& \times\left[1-2 \varepsilon \frac{k_{x}^{2}}{k_{x}^{2}+k_{z}^{2}}+2(\varepsilon-\delta) \frac{k_{x}^{2} k_{z}^{2}}{\left(k_{x}^{2}+k_{z}^{2}\right)^{2}}\right],
\end{aligned}
$$

where $k_{z}$ and $k_{x}$ are the vertical and horizontal wavenumbers. Here, the Padé coefficients $\alpha$ and $\beta$ in equation 17 of Schleicher and Costa (2015) are set to $1 / 2$ and $1 / 4$ respectively, and their coefficient $f$ is set to unity for acoustic media.

\section{OBJECTIVE FUNCTIONS IN THE IMAGE DOMAIN}

Extended images are produced by retaining the correlation lags between the source and receiver wavefields in the output. The general imaging condition can be formulated as follows (Sava and Vasconcelos, 2011):

$$
I(\mathbf{x}, \lambda, \tau)=\sum_{e, t} W_{s}(\mathbf{x}-\lambda, t-\tau) W_{r}(\mathbf{x}+\lambda, t+\tau),
$$

where $I(\mathbf{x}, \lambda, \tau)$ is the extended image, $W_{s}$ and $W_{r}$ are the source and receiver wavefields, respectively, $\lambda$ is the space lag, and $\tau$ is the time lag. Nonzero-lag energy can be used to update the migration velocity model by applying the DSO operator (Symes and Carazzone, 1991). For the horizontal space-lag extended image $I$, that operator has the form:

$$
J_{D S O}=\frac{1}{2}\left\|\lambda_{x} I\left(x, z, \lambda_{x}\right)\right\|_{\ell_{2}}^{2},
$$

where $\lambda_{x}$ is a penalty operator. Another commonly used (stackpower) objective function measures zero-lag energy:

$$
J_{S T}=\frac{1}{2}\left\|I\left(x, z, \lambda_{x}=0\right)\right\|_{\ell_{2}}^{2} .
$$

Zhang and Shan (2013) propose a "partial" stack-power objective function that combines the criteria in equations 3 and 4 
without the need to find optimal weights for $J_{D S O}$ and $J_{S T}$ :

$$
J_{P S T}=-\frac{1}{2}\left\|H\left(\lambda_{x}\right) I\left(x, z, \lambda_{x}\right)\right\|_{\ell_{2}}^{2},
$$

where $H$ is a Gaussian operator centered at zero-lag.

\section{GRADIENT COMPUTATION USING THE ADJOINT-STATE METHOD}

Here, we employ the adjoint-state method (Plessix, 2006) to obtain the gradient expressions. For most TI models, the gradients can be computed with the three leading terms of the separable dispersion relation 1 . We truncate equation 1 only for deriving the gradients and not for modeling.

\section{Data domain}

We define the data-domain model vector as $\widehat{\mathbf{m}}=\left\{V_{\text {hor }}, \eta, \varepsilon\right\}$. The gradient of the $\ell_{2}$-norm data-difference objective function is given by:

$$
\begin{aligned}
\frac{\partial J}{\partial \varepsilon}= & \sum_{e, \tau} K(\tau) \frac{2 V_{\text {hor }}^{2}}{(1+2 \varepsilon)^{2}} k_{z}^{2} u \star a \\
\frac{\partial J}{\partial \eta}= & \sum_{e, \tau} K(\tau) \frac{2 V_{\text {hor }}^{2}}{(1+2 \eta)^{2}} \frac{k_{x}^{2} k_{z}^{2}}{k_{x}^{2}+k_{z}^{2}} u \star a \\
\frac{\partial J}{\partial V_{\text {hor }}}= & -\sum_{e, \tau} K(\tau) 2 V_{\text {hor }}\left[k_{x}^{2} u \star a+\frac{k_{z}^{2} u}{1+2 \varepsilon} \star a-\right. \\
& \left.\frac{2 \eta}{1+2 \eta} \frac{k_{x}^{2} k_{z}^{2}}{k_{x}^{2}+k_{z}^{2}} u \star a\right],
\end{aligned}
$$

where $K(\tau)$ is the Dirac delta-function, and $u$ and $a$ are the forward and adjoint wavefields, respectively.

\section{Image domain}

For waveform inversion of reflection data, Alkhalifah and Plessix (2014) suggest to define the model vector as $\mathbf{m}=\left\{V_{\mathrm{nmo}}, \eta, \delta\right\}$. The adjoint sources for the partial stack-power objective function are:

$$
\left[\begin{array}{l}
g_{s} \\
g_{r}
\end{array}\right]=-\sum_{\lambda_{x}} H\left(\lambda_{x}\right)^{2}\left[\begin{array}{l}
I\left(x+\lambda_{x}, \lambda_{x}\right) u_{r}\left(x+2 \lambda_{x}, t\right) \\
I\left(x-\lambda_{x}, \lambda_{x}\right) u_{s}\left(x-2 \lambda_{x}, t\right)
\end{array}\right],
$$

where $u_{s}$ and $u_{r}$ are the source and receiver wavefields, respectively. After computing the corresponding adjoint wavefields $a_{s}$ and $a_{r}$, the source- and receiver-side gradients are found as:

$$
\begin{aligned}
\frac{\partial J}{\partial \delta}= & \sum_{e, \tau} K(\tau) \frac{2 V_{\mathrm{nmo}}^{2}}{(1+2 \delta)^{2}} k_{z}^{2} u_{i} \star a_{i} \\
\frac{\partial J}{\partial \eta}= & -\sum_{e, \tau} K(\tau) 2 V_{\mathrm{nmo}}^{2} \frac{k_{x}^{4}}{k_{x}^{2}+k_{z}^{2}} u_{i} \star a_{i} \\
\frac{\partial J}{\partial V_{\mathrm{nmo}}}= & -\sum_{e, \tau} K(\tau) 2 V_{\mathrm{nmo}}\left[k_{x}^{2} u_{i} \star a_{i}+\frac{k_{z}^{2}}{1+2 \delta} u_{i} \star a_{i}+\right. \\
& \left.2 \eta \frac{k_{x}^{4}}{k_{x}^{2}+k_{z}^{2}} u_{i} \star a_{i}\right], i=s, r,
\end{aligned}
$$

\section{SYNTHETIC EXAMPLES}

Below, we test the derived gradient expressions on two simple VTI models. The medium parameters are specified on a rectangular grid, and the density is assumed to be constant. The gradients obtained with the pseudospectral operator are compared with the ones for the space-time finite-difference (FD) alrorithm proposed by Fletcher et al. (2009); the corresponding adjoint extrapolation is developed by Wang and Sava (2015).

\section{Model 1}

First, we compute the gradients in the data domain for a model that includes a constant $V_{\text {hor-field and Gaussian anomalies in }}$ the parameters $\eta$ (reaching 0.2 at the center) and $\varepsilon$ (reaching 0.15) (Figure 1). Only transmitted waves are employed to generate parameter updates. The source function is a Ricker wavelet with a central frequency of $2 \mathrm{~Hz}$. Using the actual $\eta$ field, we compute the gradients for understated and overstated peak values of the $\varepsilon$-anomaly ( $\varepsilon=0$ and 0.3 ; the background $\varepsilon=0$ is correct). Note that for the peak frequency of the source signal $(2 \mathrm{~Hz})$ and the model size, the $\varepsilon$-errors cause data residuals that do not exceed half a cycle.

For the chosen parameterization $\left(V_{\text {hor }}, \eta, \varepsilon\right)$, the coefficient $\varepsilon$ should be constrained for near-vertical propagation, if $V_{\text {hor }}$ has been estimated from long-offset data (Alkhalifah and Plessix, 2014). We compute the gradients using the vertical ("borehole") receiver array shown in Figure 1d. In general, P-wave reflection moveout must be supplemented with borehole (Wang and Tsvankin, 2013) or other information to resolve the VTI parameters. The gradients generated by both operators are similar and, as expected, change sign depending on the sign of the $\varepsilon$-error (Figure 2). Because the background $\eta$-field is positive, the gradients produced with the FD extrapolator contain a pronounced shear-wave artifact. In the data domain, the gradient for the actual $\varepsilon$-field goes to zero. However, the datadifference estimate is questionable for real-data applications because the acoustic approximation does not accurately model P-wave amplitudes.

\section{Model 2}

Next, we compute the $\eta$-gradient in the image domain using reflection data. The model includes a horizontal interface beneath a homogeneous VTI layer with $V_{\mathrm{nmo}}=2 \mathrm{~km} / \mathrm{s}, \eta=$ $\delta=0.15$, and a thickness of $2 \mathrm{~km}$. The near-surface layer $0.2 \mathrm{~km}$-thick is assumed to be elliptic $(\varepsilon=\delta)$ to suppress the shear-wave artifact produced by the FD extrapolator. We generate horizontal-space-lag extended images (Figure 3) and obtain the $\eta$-gradients for understated and overstated values of $\eta$. The $\eta$-errors induce residual energy in extended images (Figure 3) that has a linear ("V"-like) shape, which is typical for near-horizontal interfaces (Sava and Alkhalifah, 2012; Li et al., 2015). For both extrapolators, the extended images computed with the understated and even actual $\eta$-fields also contain considerable residual energy that spreads from the image point up to the surface. These kinematic artifacts, caused by having the reflector illuminated only from the surface, may introduce bias in the image-domain objective function and lead to false model updates.

where $i$ denotes either the source or receiver side. 


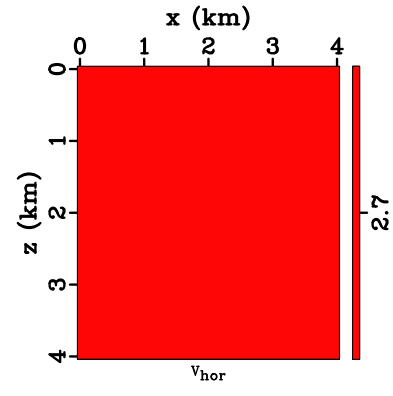

(a)

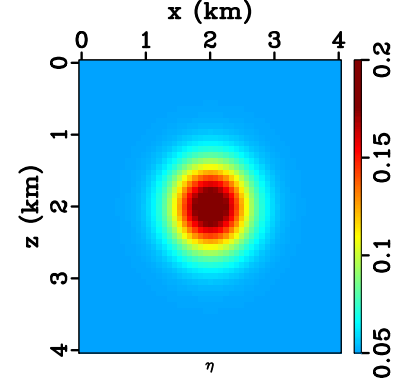

(b)

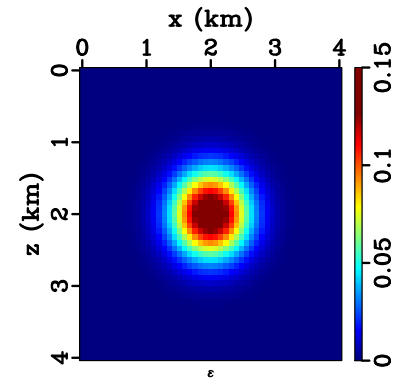

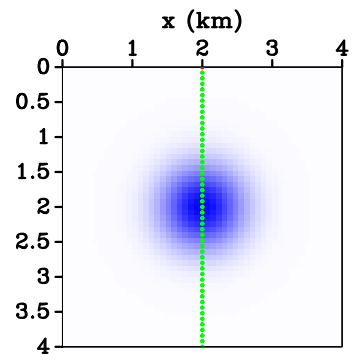

(d)

Figure 1: VTI model with Gaussian anomalies in the parameters $\eta$ and $\varepsilon$ : (a) $V_{\text {hor }}$, (b) $\eta$, and (c) $\varepsilon$ (model 1). (d) Source (red dot)-receiver (green dots) geometry.
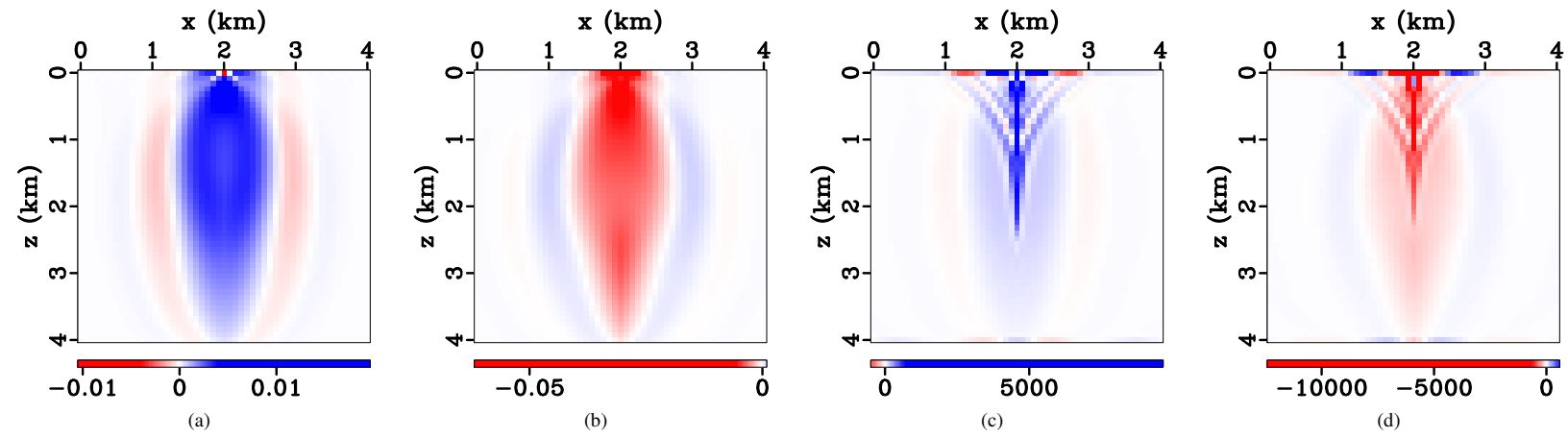

Figure 2: Gradients for model 1 computed using the (a,b) pseudospectral and (c,d) FD extrapolators with different peak values of $\varepsilon$ : $(\mathrm{a}, \mathrm{c}) \varepsilon=0$ and $(\mathrm{b}, \mathrm{d}) \varepsilon=0.3$ (the actual peak $\varepsilon=0.15$ ). The FD algorithm produces a strong artifact at $x=2 \mathrm{~km}$.

The DSO gradients computed using surface acquisition geometry and the entire extended image are shown in Figure 4. With either extrapolation operator, the gradient of the DSO objective function (equation 3 ) for the understated $\eta$-field is strongly influenced by the kinematic artifacts in the extended image. The contribution of the artifact is even larger than that of the residual induced by the $\eta$-error because the artifact is located closer to the physical sources and receivers. The partial stack-power objective function (equation 5) significantly reduces the artifact (Figure 5). Nevertheless, robust anisotropic inversion may require additional suppression of kinematic artifacts, which can be achieved by proper accounting for illumination in the imaging or DSO penalty operators (Lameloise et al., 2015; Hou and Symes, 2015; Yang and Sava, 2015).

\section{CONCLUSIONS}

We implemented forward and adjoint pseudospectral extrapolation operators based on a VTI separable dispersion-relation approximation and derived the corresponding wavefield-tomography gradients. This work is mostly focused on image-domain tomography, which is less susceptible to amplitude distortions produced by acoustic algorithms. However, because estimation of all three relevant VTI parameters (e.g., $V_{P 0}, \varepsilon$, and $\delta$ ) is seldom feasible using only $\mathrm{P}$-wave reflection moveout, we also derived data-domain gradients, which can easily incorporate borehole information. The similarity between the gradients obtained with the pseudospectral and FD operators validates our analytic results. For a model where the sources and receivers were placed in a layer with $\eta>0$, the gradients computed with the pseudospectral algorithm do not contain the imprint of the shear-wave artifact that contaminates the FD results.

The image-domain example reveals illumination-related issues with the DSO objective function applied to cross-correlation extended images. Kinematic artifacts caused by insufficient illumination substantially distort the gradients and should be suppressed prior to updating the model. The partial stackpower objective function helps reduce the false updates caused by these artifacts. Ongoing work involves implementing the imaging and inversion steps of anisotropic image-domain tomography and an extension of the algorithm to tilted TI media.

\section{ACKNOWLEDGMENTS}

This work was supported by the Consortium Project on Seismic Inverse Methods for Complex Structures at CWP and competitive research funding from King Abdullah University of Science and Technology (KAUST). 


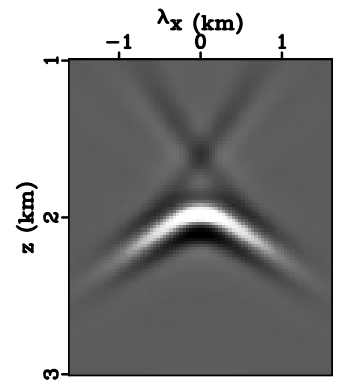

(a)

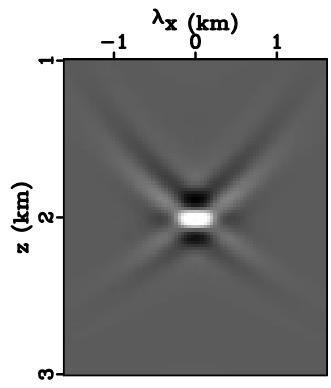

(b)

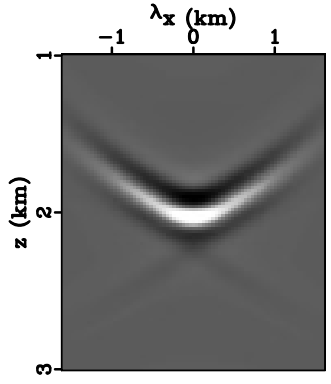

(c)

Figure 3: Space-lag CIGs for a horizontal VTI layer (model 2) computed at $x=4 \mathrm{~km}$ using the pseudospectral extrapolator with (a) $\eta=0$, (b) $\eta=0.15$ (actual value), and (c) $\eta=0.3$.
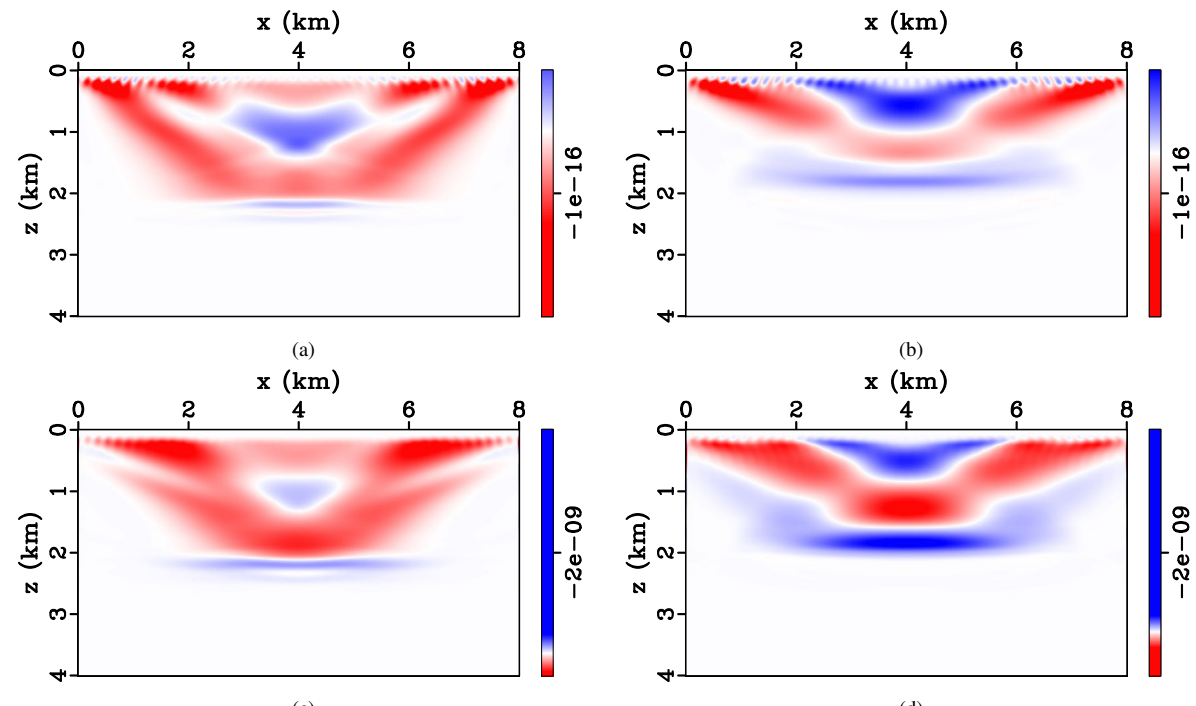

(c)

(d)

Figure 4: Gradients of the DSO objective function (equation 3) for model 2 computed using (a,b) the pseudospectral extrapolator and (c,d) the FD extrapolator for (a,c) $\eta=0$ and $(\mathrm{b}, \mathrm{d}) \eta=0.3$.
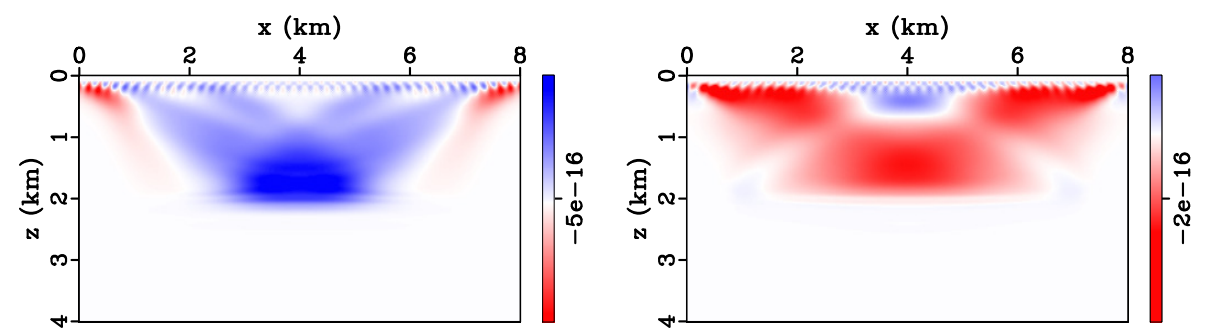

(a)

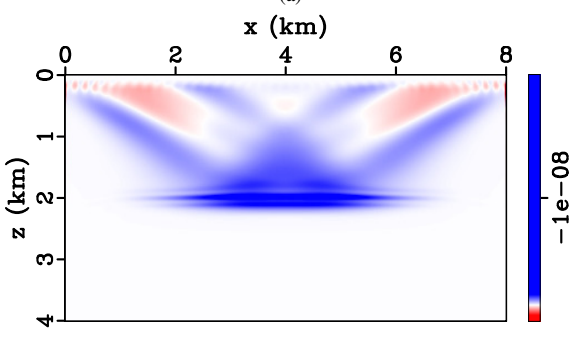

(c)

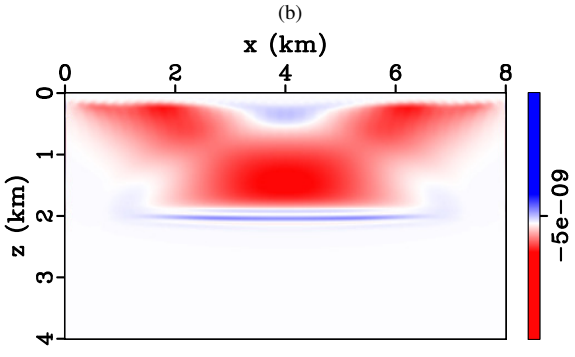

(d)

Figure 5: Gradients of the partial stack-power function (equation 5) for model 2 computed using (a,b) the pseudospectral extrapolator and (c,d) the FD extrapolator for (a,c) $\eta=0$ and (b,d) $\eta=0.3$. 
EDITED REFERENCES

Note: This reference list is a copyedited version of the reference list submitted by the author. Reference lists for the 2016 SEG Technical Program Expanded Abstracts have been copyedited so that references provided with the online metadata for each paper will achieve a high degree of linking to cited sources that appear on the Web.

\section{REFERENCES}

Al-Yahya, K., 1989, Velocity analysis by iterative profile migration: Geophysics, 54, 718-729, http://dx.doi.org/10.1190/1.1442699.

Alkhalifah, T., 1998, Acoustic approximations for processing in transversely isotropic media: Geophysics, 63, 623-631, http://dx.doi.org/10.1190/1.1444361.

Alkhalifah, T., and R.-E. Plessix, 2014, A recipe for practical full-waveform inversion in anisotropic media: An analytical parameter resolution study: Geophysics, 79, no. 3, R91-R101, http://dx.doi.org/10.1190/geo2013-0366.1.

Chavent, G., and C. A. Jacewitz, 1995, Determination of background velocities by multiple migration fitting: Geophysics, 60, 476-490, http://dx.doi.org/10.1190/1.1443785.

Du, X., P. J. Fowler, and R. P. Fletcher, 2014, Recursive integral time-extrapolation methods for waves: A comparative review: Geophysics, 79, no. 1, T9-T26, http://dx.doi.org/10.1190/geo20130115.1.

Duveneck, E., P. Milcik, P. M. Bakker, and C. Perkins, 2008, Acoustic VTI wave equations and their application for anisotropic reverse-time migration: 78th Annual International Meeting, SEG, Expanded Abstracts, 2186-2190, http://dx.doi.org/10.1190/1.3059320.

Etgen, J., and S. Brandsberg-Dahl, 2009, The pseudo-analytical method: Application of pseudoLaplacians to acoustic and acoustic anisotropic wave propagation: 79th Annual International Meeting, SEG, Expanded Abstracts, 2552-2556, http://dx.doi.org/10.1190/1.3255375.

Fletcher, R. P., X. Du, and P. J. Fowler, 2009, Reverse time migration in tilted transversely isotropic (TTI) media: Geophysics, 74, no. 6, WCA179-WCA187, http://dx.doi.org/10.1190/1.3269902.

Hou, J., and W. W. Symes, 2015, An approximate inverse to the extended born modeling operator: Geophysics, 80, no. 6, R331-R349, http://dx.doi.org/10.1190/geo2014-0592.1.

Lameloise, C.-A., H. Chauris, and M. Noble, 2015, Improving the gradient of the image-domain objective function using quantitative migration for a more robust migration velocity analysis: Geophysical Prospecting, 63, 391-404, http://dx.doi.org/10.1111/gpr.2015.63.issue-2.

Li, V., I. Tsvankin, and T. Alkhalifah, 2015, Analysis of RTM extended images for VTI media: 85th Annual International Meeting, SEG, Expanded Abstracts, 519-524.

Li, Y. E., B. Biondi, D. Nichols, and R. Clapp, 2016, Toward a closed loop from seismic imaging to earth-model building: The Leading Edge, 35, 135-139, http://dx.doi.org/10.1190/tle35020135.1.

Pestana, R., B. Ursin, and P. L. Stoffa, 2011, Separate P- and SV-wave equations for VTI media: 81st Annual International Meeting, SEG, Expanded Abstracts, 163-167, http://dx.doi.org/10.1190/1.3627518.

Plessix, R.-E., 2006, A review of the adjoint-state method for computing the gradient of a functional with geophysical applications: Geophysical Journal International, 167, 495-503, http://dx.doi.org/10.1111/gji.2006.167.issue-2.

Rickett, J., and P. Sava, 2002, Offset and angle-domain common image-point gathers for shot-profile migration: Geophysics, 67, 883-889, http://dx.doi.org/10.1190/1.1484531.

Sava, P., 2014, A comparative review of wavefield tomography methods: CWP Project Review Report, 119-144.

Sava, P., and T. Alkhalifah, 2012, Anisotropy signature in extended images from reverse-time migration: 82nd Annual International Meeting, SEG, Expanded Abstracts, 1-6, http://dx.doi.org/10.1190/segam2012-0124.1. 
Sava, P., and S. Fomel, 2006, Time-shift imaging condition in seismic migration: Geophysics, 71, no. 6, S209-S217, http://dx.doi.org/10.1190/1.2338824.

Sava, P., and I. Vasconcelos, 2011, Extended imaging conditions for wave-equation migration: Geophysical Prospecting, 59, 35-55, http://dx.doi.org/10.1111/gpr.2010.59.issue-1.

Schleicher, J., and J. C. Costa, 2015, A separable stronganisotropy approximation for pure qp wave propagation in TI media: 85th Annual International Meeting, SEG, Expanded Abstracts, 685, 3565-3570.

Symes, W.W., and J. J. Carazzone, 1991, Velocity inversion by differential semblance optimization: Geophysics, 56, 654-663, http://dx.doi.org/10.1190/1.1443082.

Tsvankin, I., 2012, Seismic signatures and analysis of reflection data in anisotropic media, 3rd ed.: SEG.

Wang, H., and P. Sava, 2015, Pseudo-acoustic wavefield tomography with model constraints: CWP Project Review Report, 95-106.

Wang, X., and I. Tsvankin, 2013, Multiparameter TTI tomography of P-wave reflection and VSP data: Geophysics, 78, no. 5, WC51-WC63, http://dx.doi.org/10.1190/GEO2012-0394.1.

Weibull, W. W., and B. Arntsen, 2014, Anisotropic migration velocity analysis using reverse-time migration: Geophysics, 79, no. 1, R13-R25, http://dx.doi.org/10.1190/geo2013-0108.1.

Yang, T., and P. Sava, 2015, Image-domain wavefield tomography with extended common-image-point gathers: Geophysical Prospecting, 63, no. 5, 1086-1096, http://dx.doi.org/10.1111/13652478.12204.

Zhang, Y., and G. Shan, 2013, Wave-equation migration velocity analysis using partial stack-power maximization: 83rd Annual International Meeting, SEG, Expanded Abstracts, 4847-4852, http://dx.doi.org/10.1190/segam2013-0716.1.

Zhang, Y., H. Zhang, and G. Zhang, 2011, A stable TTI reverse time migration and its implementation: Geophysics, 76, no. 3, WA3-WA11, http://dx.doi.org/10.1190/1.3554411. 